\title{
Sécurisation des parcours professionnels et flexicurité : analyse comparative des positions syndicales
}

Job security and Flexicurity: a comparison of the French trade unions stances

\section{Solveig Grimault}

\section{(2) OpenEdition}

\section{Journals}

Édition électronique

URL : http://journals.openedition.org/travailemploi/2350

DOI : 10.4000/travailemploi.2350

ISSN : 1775-416X

Éditeur

DARES - Ministère du Travail

Édition imprimée

Date de publication : 17 mars 2008

Pagination : 75-89

ISSN : 0224-4365

\section{Référence électronique}

Solveig Grimault, « Sécurisation des parcours professionnels et flexicurité : analyse comparative des positions syndicales », Travail et Emploi [En ligne], 113 | janvier-avril 2008, mis en ligne le 17 février 2011, consulté le 19 avril 2019. URL : http://journals.openedition.org/travailemploi/2350 ; DOI :

10.4000/travailemploi.2350 


\title{
Sécurisation des parcours professionnels et flexicurité: analyse comparative des positions syndicales
}

\author{
Solveig Grimault $\left.{ }^{*}\right)$
}

L'analyse comparative des positions des organisations syndicales en matière de flexicurité et de sécurisation des parcours professionnels fait ressortir la forte spécificité de leurs différentes positions ainsi que la correspondance, qui perdure, entre les propositions respectives des organisations et la place qu'elles occupent les unes par rapport aux autres dans l'équilibre général du système de relations professionnelles. L'auteure propose un schéma représentant les "frontières du travail salarié», de plus en plus poreuses, selon des catégories élaborées par A. Supiot à propos des transformations du travail. Elle localise les différents syndicats dans ce cadre, par rapport à deux axes de recomposition possibles du droit du travail: la question de l'acquisition d'un statut protecteur et la question des transitions et de leur prise en charge. Elle formule des hypothèses pour expliquer la continuité des positions mises en évidence et évoque des pistes de réflexion qui n'ont pas été développées, faute d'un véritable processus de concertation en amont.

L'article se propose d'analyser les positions des organisations syndicales françaises relatives à la flexicurité, à la lumière des différents concepts qu'elles mobilisent sur le thème de la sécurisation des parcours professionnels. Deux sources ont fortement contribué à structurer les débats sur la sécurisation des parcours professionnels - le rapport Boissonnat (CGP, 1995) et le rapport Supiot (SUPIOT, 1999). Ces deux rapports sont à l'arrière-plan des réflexions des organisations syndicales sur ce thème, qu'elles s'en démarquent ou qu'elles s'en inspirent. Cet article revient, dans un premier temps, sur cette généalogie des positions syndicales. Nous présentons ensuite les positions syndicales sur la sécurisation des parcours professionnels, non pas de façon exhaustive, mais en mettant en évidence l'intention principale, l'orientation ou l'axe que chaque organisation privilégie aujourd'hui sur ce thème. Quoique centré sur l'analyse des positions syndicales, l'article fournira, sur certains points particuliers, des éléments permettant de situer la position des organisations d'employeurs - Medef et CGPME en particulier. Enfin, l'article propose une sorte de «cartographie» des positions syndicales, afin de mieux distinguer les approches des uns et des autres.

Cette «mise à plat» met en évidence une «homologie structurale» entre la position qu'occupe chaque organisation dans le champ des relations professionnelles, et les propositions ou le modèle qu'elle défend sur le thème de la flexicurité et de la

(*) Chercheure à 1'Institut de recherches économiques et sociales. Solveig.GRIMAULT@ires-fr.org. sécurisation des parcours professionnels. Les prises de positions syndicales s'analysent ainsi comme la rencontre entre un ensemble de «modèles» disponibles (règles, institutions, etc. ), et les dispositions propres à chaque organisation, ce à quoi chacune est prête. À ce jour, le peu de concertation en amont et les modalités d'agencement des débats collectifs n'ont pas encore véritablement permis de faire émerger de nouveaux objets, susceptibles de décliner le thème de la sécurisation des parcours professionnels. Ce dernier, dans certaines propositions syndicales du moins, met pourtant explicitement en jeu des pratiques et des dimensions qui, pour certaines, participent directement de la structure même du champ des relations professionnelles: modes d'institutionnalisation du travail, modalités d'acquisition d'un statut et conditions de la mobilité statutaire pour les personnes, nécessité de construire de nouvelles garanties collectives comme soutien à l'autonomisation des individus, reformulation de l'articulation entre travail et protection sociale.

\section{Les débats sur la flexicurité en France: deux rapports et peu de concertation}

En 1995, le rapport de la commission Boissonnat du Commissariat général du Plan propose une lecture des mutations du travail, et envisage de nouveaux types de compromis possibles dans les relations d'emploi. Il introduit ainsi ce nouvel objet qu'est la sécurisation des parcours professionnels, et fait du 
«contrat d'activité» son principal outil. Quelques années plus tard, les travaux menés par le groupe d'experts européens réunis autour d'Alain Supiot se réclament, de la même façon, d'une «approche compréhensive» des évolutions du travail. Face à un modèle social considéré comme à bout de souffle, les auteurs avancent l'idée d'un nouvel «état professionnel» des personnes, susceptible de prendre en compte la diversité des formes de travail expérimentées tout au long de la vie humaine-emploi, formation, travail indépendant, travail hors marché. À cet état professionnel répondrait un nouvel agencement des droits sociaux, pensés selon des cercles concentriques (droits universels attachés à la personne, droits fondés sur le travail non professionnel, droits attachés à l'exercice d'une activité professionnelle, droits liés à une situation particulière de travail salarié). On retrouve dans les premières réflexions syndicales sur la sécurisation des parcours professionnels la marque de ces deux sources, qui font référence dans les débats. On note, d'une part, que l'un des traits communs des premières réflexions syndicales sur ce thème est l'importance notable accordée au travail et à ses évolutions. D'autre part, l'idée s'impose progressivement de conférer un nouveau statut à la personne, déconnecté de l'emploi, dans l'architecture des droits sociaux. La notion de «droits attachés à la personne garantis collectivement dans le cadre d'institutions chargées de leur mise en œuvre», sera ainsi la traduction de la CGT des propositions du rapport Supiot.

La CGT introduit ainsi la revendication d'une nécessaire refondation des droits sociaux, innovation qu'elle place au même rang que l'invention, naguère, des conventions collectives ou de la sécurité sociale, et qui devrait déboucher sur «un nouveau statut du travail» (LE Duigou, 2002, p. 56). De là vient le Nouveau statut du travail salarié (NSTS), dont la confédération a fait, depuis, l'un de ses principaux axes revendicatifs. Nouvel horizon en terme de garanties collectives, conçu comme un socle de droits interprofessionnels communs, attachés à tous les salariés, ce nouveau statut vise "à sécuriser les parcours professionnels en terme de rémunération, de formation, de droits, de liberté, de retraite, de protection sociale, quels que soient les aléas de la carrière et la profession» (CGT, 2003). Ces nouveaux droits sont conçus comme «transférables pour chaque salarié au fur et à mesure de ses changements d'emplois, en cas de changement d'entreprise (1)» et en période de chômage. Le Nouveau statut du travail salarié figure, dans le document d'orientation du congrès d'avril 2006 dans la partie sur la démocratie: l'enjeu est bien de repenser l'architecture de la démocratie sociale.

(1) «Négociation sur le marché du travail. Au pas de charge», La Nouvelle Vie Ouvrière, 26 octobre 2007, p. 12.
La CFDT, quant à elle, retient du rapport Boissonnat l'idée qu'il s'agit de «rénover en profondeur le cadre institutionnel et juridique relatif au travail» (KARVAR, 2005, p. 53). Le rapport Supiot aura ensuite permis d'envisager la manière dont pourrait se trouver «rétablie une continuité des droits à travers la discontinuité des trajectoires professionnelles (...) en transférant les droits du statut de l'emploi à la personne du travailleur» (ibid.). La CFDT précise toutefois, d'emblée, que si «des droits à la formation, à la santé, au conseil en parcours professionnel, individuels et transférables tout au long de la vie, ont [ainsi] trouvé leur place dans l'agenda social, leur instauration ne relève pas de l'octroi unilatéral d'un statut mais d'une construction progressive par la négociation collective et le compromis » (ibid.). La confédération énonce alors les voies qu'elle privilégiera en matière de sécurisation des parcours professionnels : la négociation et l'identification de la formation comme «enjeu syndical et social essentiel», «enjeu économique de première importance» dans une économie mondialisée.

On retrouve enfin, dans l'ouvrage collectif que la CFTC consacre à «la sécurisation des parcours de vie », la façon dont les travaux autour de Boissonnat et Supiot ont pu nourrir sa réflexion. Le statut du travailleur édité en avril 2006 par la CFTC (SIMON, 2006), formalise un ensemble de propositions visant à «harmoniser et sécuriser tous les temps de vie professionnels et extraprofessionnels ainsi que leurs aléas ». Fidèle à la morale sociale-chrétienne, la CFTC y revendique le respect de la personne, dans sa globalité et tout au long de sa vie, et plaide pour une conception élargie du travail qui «englobe les salariés, les travailleurs indépendants mais aussi les personnes occupant des activités à utilité sociale telles que le travail parental et l'engagement dans le bénévolat» pour concevoir un ensemble de droits nouveaux, accessibles à tous. L'ouvrage propose ainsi une amélioration des dispositifs en vigueur, sous la forme d'un socle de droits sociaux minimaux garantis quelle que soit la situation de la personne.

Les rapports Boissonnat et Supiot n'ont naturellement pas été les seules sources alimentant les propositions syndicales (2). Mais elles les ont marquées d'une façon singulière. Le rapport Boissonnat, on l'a dit, a défini ce nouvel objet qu'est la sécurisation des trajectoires. En cela il constitue un point de référence dans les débats. Contrairement à d'autres rapports du Commissariat général du Plan et en dépit de la tradition de concertation dont cet organisme, alors,

(2) La CGT revendique, en particulier, la référence à Paul Boccara, (1996, p. 9-105). Les écrits de la CGT et de la CFDT font par ailleurs également référence, parmi d'autres, aux travaux de Marie-Laure Morin, ou encore, pour la CGT, à ceux de Robert Salais - membre par ailleurs du groupe d'experts réunis autour de Supiot-, Bruno Trentin, Bernard Friot et, pour la CFDT, aux ouvrages de Bernard Gazier et Robert Castel notamment. 
était porteur, ce rapport n'est cependant pas le fruit des travaux d'une commission de concertation associant les partenaires sociaux. Il n'a pas, de ce fait, donné lieu à un travail de problématisation collective du thème de la sécurisation des trajectoires. En effet, dès lors que "problématiser un thème, c'est définir collectivement les "points de passage obligés" de la réflexion collective pour le traiter» (Duclos, Mirandon, 2003, p. 5), cela nécessite un agencement particulier des débats qui, en l'occurrence, n'a pas été recherché - le rapport Supiot en revanche n'avait pas vocation à structurer une telle démarche. L'absence de processus de concertation n'aura donc pas permis, dans un premier temps au moins, la problématisation collective du thème de la sécurisation des trajectoires. Les rapports, dans cette configuration, ne viennent pas "s'interposer avec une "question nouvelle" dans le jeu réglé du partenariat social [ou de] la confrontation sociale» (ibid.); ils ne contribuent pas à «agencer un débat en construisant son objet propre», mais deviennent, classiquement, une source partagée que chacun des acteurs mobilise à son gré.

Les travaux menés peu de temps après par le Commissariat général du Plan sur «les perspectives de la France»(CGP, 2000), dans le cadre d'une commission de concertation, ne changent pas fondamentalement la donne. Le thème de la sécurisation des parcours professionnels y est abordé, parmi beaucoup d'autres bien sûr. Il est cependant bien présent dans plusieurs des avis produits par les organisations syndicales à l'issue de ce travail. Les positions syndicales exprimées en fin de rapport témoignent d'une reconnaissance du travail de synthèse effectué, pour développer ensuite la perception autonome, politique, de chaque organisation. Les avis, autrement dit, ne sont, là encore, pas nés dans le débat. Ceux de leurs passages qui sont en rapport avec le thème de la sécurisation des trajectoires n'échappent pas à ce constat: on y retrouve, pour chacune des organisations qui a entamé une réflexion spécifique sur ce thème, l'orientation déjà privilégiée et évoquée ci-dessus. On comprend, à la lecture de ces avis, que le déficit de problématisation collective perdure. Les organisations d'employeurs, quant à elles, n'investissent pas encore la question à ce moment. La CGPME, quoique membre de la commission de concertation du Plan, ne formalise d'ailleurs pas d'avis dans le cadre de ce rapport.

\section{Les principales propositions syndicales sur la sécurisation des parcours professionnels}

Les propositions syndicales sur la sécurisation des parcours professionnels ne seront pas présentées ici de façon exhaustive, mais en mettant en évidence l'intention principale, l'orientation ou l'axe que chaque organisation privilégie aujourd'hui sur ce thème.

\section{Encadré 1 \\ Corpus et méthodologie}

Le corpus analysé est constitué des documents d'orientation et des résolutions des congrès confédéraux, des articles publiés dans les revues syndicales, des newsletters et textes mis en ligne sur les sites des confédérations, de documents et de notes de travail internes, des déclarations et des auditions effectuées dans le cadre d'avis et de rapports publics (déclarations des organisations relatives à l'Avis du Conseil économique et social sur la sécurisation des parcours professionnels (1), comptes rendus des auditions réalisées auprès des différentes confédérations par la mission d'information du Sénat sur le fonctionnement des dispositifs de formation professionnelle (2)). Ces sources écrites ont par ailleurs été complétées, pour chaque confédération, par un entretien réalisé auprès du responsable ou du référent chargé du dossier de la sécurisation des parcours professionnels.

Du fait de sa relative faiblesse quantitative, et de son caractère hétérogène, le corpus disponible ne se prête pas à une analyse quantitative, par exemple de type lexical. La méthodologie retenue est donc celle d'une analyse sémantique des corpus syndicaux. Le choix des mots témoigne en effet des stratégies institutionnelles déployées par les acteurs. Le fait, par exemple, que les organisations travaillent à donner des définitions plus exactes des termes mobilisés, témoigne de la nature de leurs préoccupations. Sur cet objet encore flou qu'est la sécurisation des parcours professionnels, les organisations sont, plus encore que sur d'autres terrains sans doute, investies dans une bataille sur le sens, qui s'engage donc aussi par une "lutte contre les mots (3) ». II importe donc de mettre en évidence les champs sémantiques privilégiés par chaque organisation, de rendre compte des proximités de sens que chacune établit entre les différents concepts qu'elle mobilise, afin de mieux spécifier les différentes positions en présence.

(1) Consell ÉCONomique et SOCiAL (2007).

(2) CARLe, Seiller (2007).

(3) BOURDieu (1987, p. 69).

\section{La revendication de la CGT}

d'une sécurité sociale professionnelle

La sécurité sociale professionnelle, intégrée au Nouveau statut du travail salarié, s'est d'abord imposée comme un outil de lutte contre les restructurations, tout en ayant d'emblée l'ambition d'être l'amorce d'une refonte plus générale. Maryse Dumas, secrétaire confédérale chargée de ces questions, rappelait ainsi la teneur du projet: "C'est à [l'insécurité sociale] que veut s'attaquer la proposition de la CGT : notre proposition consiste en effet à ce que tout salarié soit assuré que, même lorsque son entreprise ferme ou délocalise, son contrat de travail, lui, soit pérennisé. Il s'agirait de créer un système assis sur les cotisations sociales des entreprises et sur lequel 
pourrait converger la réaffectation des aides actuelles à l'emploi afin de financer une mesure destinée à protéger le salarié en attendant qu'il puisse être réembauché, à des conditions lui convenant et au moins équivalentes au dernier emploi occupé. Le terme "sécurité sociale professionnelle" fait ainsi référence à la fois au type de financement (par cotisations), et à la vocation (la protection du début à la fin de la vie) de la Sécurité sociale, créée à la Libération, et qui a d'ores et déjà pour mission de pallier diverses situations de non-travail en protégeant les droits des salariés» (DumAs, 2002, p. 39-40).

Le contenu de la notion de sécurité sociale professionnelle s'est progressivement précisé même si, pour la CGT, le Nouveau statut du travail salarié et la sécurité sociale professionnelle n'ont pas vocation à formaliser «un programme revendicatif prêt à l'emploi, utilisable en toutes circonstances: nous ne sommes pas dans du "prêt-à-porter" mais dans du "cousu mains", du "cas par cas"» (GABRIEL, 2006), rappelle volontiers la CGT. Le document d'orientation du congrès (3) précise que la sécurité sociale professionnelle doit permettre «que les salariés n'aient pas à souffrir des transitions entre deux emplois que leur imposent les restructurations, délocalisations ou fermetures d'entreprises dictées par les stratégies économiques et industrielles. Le contrat de travail et la rémunération doivent être maintenus jusqu'à ce que le salarié ait obtenu un reclassement effectif dans un nouvel emploi de niveau au moins équivalent au précédent. Cela doit permettre au salarié de bénéficier de toutes les conditions financières, matérielles, de disponibilité de temps, lui permettant de s'engager dans la formation de son choix»». Concernant le financement, le document se poursuit en indiquant que «les entreprises de la branche ou du bassin d'emploi doivent être mises à contribution, la solidarité organisée entre les branches, au travers de la mise en place d'un fonds mutualisé. La réorientation des fonds publics en faveur de l'emploi complète ce dispositif». La sécurité sociale professionnelle est donc pensée comme une protection pour chaque salarié tout au long de sa vie professionnelle, avec pour principe la continuité du contrat de travail. Il faut souligner ici l'importance, pour la CGT, des travaux de Bernard FRIOT (1998), qui a construit un idéal type du rapport salarial, à travers son histoire, en proposant un couplage entre emploi et protection sociale. Cette question importante a également été soulevée par Alain SuPIOT (1995). La proposition de la CGT vise en quelque sorte une extension du modèle.

Beaucoup de questions demeurent et sont en cours de discussion à la CGT, notamment sur le mode de financement et l'opérationnalisation de ce droit. Certains insistent notamment sur la nécessité, par ailleurs affirmée par le $48^{\mathrm{e}}$ congrès, de renforcer le

(3) «Document d'orientation», Le Peuple, supplément au $\mathrm{n}^{\mathrm{o}} 1632$, juin 2006 , p. 174-210. dialogue social territorial, considérant que celui-ci «doit émerger comme véritable espace de la démocratie sociale» (Le Duigou, 2007, p. 33) et qu'il est un lieu d'innovations sociales possibles dans l'accession à un nouveau statut du travail salarié, peutêtre plus que la branche professionnelle(4).

\section{La CFDT : le primat donné à la négociation pour une meilleure articulation des dispositifs de sécurisation}

À la veille de son congrès (juin 2006), la CFDT présentait dans ces termes sa position générale sur la sécurisation des parcours professionnels: «La sécurisation des parcours ne peut être dissociée de démarches d'anticipation, comme la gestion prévisionnelle des emplois et des compétences. (...) Sécuriser (...) signifie aussi garantir une continuité des droits, en dépit des ruptures auxquelles sont confrontés les individus (...). Puisque les carrières se déroulent de plus en plus en dehors d'une seule entreprise, les nouveaux droits doivent être attachés aux individus et non plus au contrat de travail. (...) Contrairement à la CGT (....), la CFDT n'entend pas sécuriser le contrat, mais bien les trajectoires professionnelles. Il n'est pas question de surprotéger le salarié, mais de faire en sorte qu'il soit acteur de son parcours. La responsabilité est partagée entre l'entreprise - dans la mise en place d'une politique de ressources humaines fondée sur l'anticipation et le développement des compétences - et les pouvoirs publics - par l'impulsion et la garantie de parcours sécurisés pour tous, avec les partenaires sociaux et les salariés $(5) \gg$. La confédération a réaffirmé, lors de son congrès, les grandes orientations de sa stratégie revendicative. L'obtention de «nouvelles garanties collectives favorisant et sécurisant les parcours professionnels pour tous les salariés (6)» constitue, d'emblée, l'un des axes prioritaires de sa stratégie syndicale. Une série de revendications, relatives à la sécurisation de «la mobilité dans l'emploi», viennent ainsi détailler le cœur des

(4) Citons, sur ce point: «L'évolution du lien de subordination conduit (...) à construire des droits sociaux et salariaux qui ne soient plus exclusivement conditionnés par l'entreprise ou la branche professionnelle du salarié, exclusive synonyme de mise en concurrence, d'inégalités et de divisions. À l'instar de ce qui existe (schématiquement) pour environ 5 millions de fonctionnaires et d'agents sous statut, les 23 millions de salariés seraient dotés de droits leur appartenant, transférables et opposables à tout employeur privé ou public. Par exemple la sécurité de l'emploi, garantie notamment par l'obligation de reclassement sur un poste équivalent, et le versement intégral de la rémunération dont bénéficient ces 5 millions de salariés du public, seraient étendus à l'ensemble des salariés » (DonNEDDU, 2006, p. 43).

(5) Céline Collot, secrétaire confédérale chargée de la sécurisation des parcours professionnels, citée dans CFDT Magazine, $\mathrm{n}^{\circ} 322$, avril 2006, p. 8.

(6) Sauf indication contraire, les expressions et extraits entre guillemets sont tirés de la résolution générale du congrès («Résolution "Engagés dans une société en mutation"», Syndicalisme hebdo, $\mathrm{n}^{\mathrm{0}} 3083$, juillet 2006, p. 62-91). 
propositions de la CFDT en matière de sécurisation des parcours professionnels.

Face au développement croissant des mobilités non choisies, mais également face aux difficultés rencontrées par les salariés dans les mobilités professionnelles choisies, la CFDT revendique «la mise en place, à tous les niveaux, de dispositifs [permettant aux salariés de] conserver une partie des droits acquis dans leurs entreprises et de disposer de moyens les aidant dans ces mobilités». Ces dispositifs devraient notamment viser à organiser la transférabilité des droits acquis dans une entreprise par la mise en place des systèmes de mutualisation nécessaires, permettre la continuité des protections sociales complémentaires, instaurer un système d'équivalence interprofessionnel de droits entre les différentes branches professionnelles, permettre la formalisation des acquis en matière de compétences et de qualifications (développement de la VAE, amélioration du DIF), étendre la transférabilité du DIF. Cette dernière, en particulier, «suppose une mutualisation des fonds, une articulation des politiques de branches et de territoires(7)». De façon générale, la CFDT poursuit les orientations de la loi du 4 mai 2004 qui a notamment repris - et créé - l'une des propositions anciennes de la confédération, le passeport formation(8). De là vient notamment l'idée de parcours(9). Notons que la CFDT est en cela - contrairement à la CGT davantage du côté de la «sécurité de l'emploi» que de la «stabilité de l'emploi», selon les distinctions établies par le rapport du Cerc(10), tout en insistant sur le nécessaire «engagement des entreprises qui doivent, en matière d'anticipation, prendre leur responsabilité notamment dans la qualification de leurs salariés(11)» et dans le maintien de cette qualification.

(7) Audition de $\mathrm{M}^{\mathrm{me}}$ Annie Thomas, secrétaire nationale CFDT et de M. Jean-Claude Gueudet, secrétaire confédéral CFDT, in CARLe, Seillier, op. cit., 2007, Tome II, p. 155.

(8) Sur la revendication d'un passeport formation, voir: http://www.cfdt.fr/actualite/formation/professionnelle/ dossier reforme form pro $02 . \mathrm{htm}$.

(9) «Chaque salarié doit être en mesure d'identifier et de faire certifier ses connaissances, ses compétences et ses aptitudes professionnelles, acquises dans le cadre de la formation initiale ou continue, ou du fait de ses expériences professionnelles. $\mathrm{Ce}$ document témoigne du parcours du salarié et permet de favoriser sa mobilité interne et externe» (CFDT, «Définir un projet professionnel. Le passeport formation », sur http://www.cfdt. $\mathrm{fr} /$ pratique/guides_droits/formation/passeport_formation.htm). (10) CERC, 2005. Le rapport du Cerc définit la «sécurite de l'emploi» comme le fait, pour une personne, de rester employée, sans interruption durable, même s'il y a changement d'entreprise; la «stabilité de l'emploi» est définie comme la continuité du lien d'emploi entre un salarié et une entreprise. Certains auteurs insistent notamment sur la façon dont les organisations syndicales se distribuent selon les différents types de sécurité formalisés par les socio-économistes du travail en Europe (Duclos, Kerbourc'h, 2006).

(11) Déclaration du groupe de la CFDT, in Conseil économique et social, op. cit., 2007, p. I-43.
Le contrat de travail est l'un des leviers sur lesquels la CFDT propose d'agir: il est nécessaire, indique le projet de résolution, «de rénover le principe et le contenu du contrat de travail dont la base doit rester à durée indéterminée». La CFDT revendique notamment d'inscrire dans le contrat de travail le principe d'une transférabilité des droits acquis par le salarié dans une entreprise, lorsque le salarié quitte l'entreprise mais également la branche professionnelle dont elle relève, et ce tout au long de sa vie professionnelle (y compris dans les phases de rupture) : en pratique, la transférabilité devrait au moins concerner le droit à la formation professionnelle continue et à l'épargne salariale.

Pour sécuriser les parcours entre deux contrats de travail, la CFDT s'inscrit dans les orientations affirmées par l'Unedic et mise notamment sur la formation(12). Ces orientations devraient être mises en œuvre dans le cadre d'un service public de l'emploi rénové, au moyen d'un rapprochement opérationnel de l'Unedic et de l'ANPE, en associant également des organismes spécialisés (Afpa). Cette structure commune placerait le demandeur d'emploi au centre du système, avec un système d'information unique, un guichet unique, un dossier unique et un référent unique. Dans cette nouvelle organisation, les partenaires sociaux conserveraient la gestion paritaire de l'indemnisation du chômage, l'État assurant quant à lui le pilotage du service public de l'emploi, et en particulier de l'accompagnement des chômeurs. Enfin, la CFDT considère qu'il conviendrait aujourd'hui, plus généralement, de «repenser le fonctionnement du paritarisme, le rôle de l'État et le lien entre solidarité nationale et solidarité salariale ».

\section{La CGT-FO: l'exigence de la prise en charge collective du risque}

La CGT-FO aborde la problématique de la sécurisation des parcours en la mettant au croisement des stratégies revendicatives qu'elle privilégie par ailleurs. Elle s'en est récemment expliquée dans ces termes: «Le DIF préfigure l'évolution du droit du travail: la naissance de nouveaux droits non plus attachés temporairement à un contrat de travail mais constituant une forme de viatique accompagnant le salarié dans son parcours professionnel. Il n'est pas question de faire de la formation professionnelle la pierre philosophale qui résoudrait tous les problèmes. Mais il en va du concept de "Sécurisation des parcours professionnels" comme de la "Formation tout au long de la vie". D'une expression creuse et convenue, nous devons faire un

(12) $C f$. sur ce point: «Il faut sécuriser les parcours entre deux contrats de travail. Il n'est pas logique, par exemple, que ce soient les chômeurs qui aient le moins accès à la formation professionnelle» (Annie Thomas, secrétaire nationale, citée dans Syndicalisme hebdo, no 3073, avril 2006). 
espace de revendications» (Quentin, 2006). Pour FO, la question de la sécurisation des parcours est donc avant tout à la croisée de trois champs d'intervention: la formation professionnelle, les régimes d'assurance chômage et le traitement des restructurations. C'est donc avant tout dans chacun de ces domaines que des contenus viables et susceptibles de donner corps à l'idée de sécurisation doivent être négociés. Concernant les deux premiers champs de négociation que sont la formation et la convention assurance chômage, FO insiste sur les avancées qu'ont notamment constituées l'introduction d'une définition juridique du plan de formation dans les entreprises, dans le cadre de l'accord sur le DIF, ou encore la création de dispositifs tels que le contrat et la période de professionnalisation. La confédération reste cependant réservée sur les bénéfices qui peuvent être attendus de la seule formation, et se refuse à toute focalisation qu'elle jugerait excessive sur cette dimension. Rappelant par ailleurs son attachement au système paritaire, la CGT-FO est opposée à «toute fusion Unedic/ANPE et à toute forme de tripartisme (13)». Elle milite par ailleurs pour une contractualisation «entre organismes paritaires au niveau national (et) avec les autres parties prenantes $(14) »$ au niveau territorial (constitution, par exemple d'un "pool paritaire, interlocuteur du conseil régional en matière de formation continue et de reclassement des demandeurs d'emploi(15)»).

Plus fondamentale est, du point de vue de la CGTFO, l'exigence de mutualisation. La prise en charge collective du risque constitue en effet, pour FO, le premier élément de sécurisation des salariés dans le traitement des restructurations. C'est la raison pour laquelle $\mathrm{FO}$ revendique notamment la création d'un fonds de mutualisation destiné à assurer pendant au moins un an le contrat de travail des salariés victimes d'un plan social, en y incluant également les salariés des entreprises sous-traitantes dont l'état de dépendance au donneur d'ordre touché par la restructuration est avéré. Le maintien du lien contractuel avec l'entreprise est en effet considéré comme essentiel pour permettre à un «droit d'initiative du salarié dans sa reconversion $(16) »$ de s'exercer, quelle que soit la situation de l'entreprise. C'est également au moyen de la mutualisation que FO envisage la transférabilité du DIF : elle insiste ainsi sur «l'importance de la notion de droits acquis dans le cadre

(13) «Résolution du Comité confédéral national de la CGFFO, Paris, 7 et 8 mars 2007», FO Hebdo, no 2795, p. 14.

(14) «Déclaration du groupe de la CGT-FO», in ConseIL ÉCONOMIQUE ET SOCIAL, 2007, op. cit., p. I-51.

(15) Audition de M. Jean-Claude Quentin, secrétaire confédéral chargé de l'emploi, de la formation professionnelle et de l'assurance chômage, M. Christophe Couillard et M. Youcef Tayeb, assistants confédéraux, CGT-FO, in CARLE, SeIllier, 2007, op. cit., Tome II, p. 128.

(16) «Cohésion sociale \& accompagnement des mutations économiques», Force Ouvrière, Bulletin Emploi, $\mathrm{n}^{\mathrm{o}} 4$, janvier 2005 . du contrat de travail, attaché à la personne et garanti collectivement par la mutualisation, (...) l'assurance chômage (illustrant), d'une certaine façon, cette $\operatorname{association}(17) »$.

\section{La CFTC: des éléments de formalisation d'un projet de société au service de la sécurisation des parcours de vie}

Les propositions - non exhaustives, précise la CFTC - s'étendent sur une large palette de protections et de droits sociaux. Nous présentons ici les principales mesures.

En matière de formation, le statut du travailleur prévoit que tout jeune sortant du système scolaire bénéficie d'un crédit individuel de formation inversement proportionnel à la durée de ses études. Ce crédit alimenterait un chèque formation, abondé ensuite tout au long de sa vie professionnelle. Ce droit, attaché à la personne, serait transférable d'une entreprise à une autre.

La CFTC fait un certain nombre de propositions visant à encadrer le travail précaire et le temps partiel contraint. Elle prévoit notamment une majoration des cotisations employeur d'assurance chômage pour les contrats précaires (CDD, intérim) lorsque ceux-ci dépassent un certain pourcentage de l'effectif global, une meilleure indemnisation chômage des titulaires de contrats courts, le développement des groupements d'employeur - qui n'embaucheraient qu'en CDI à temps plein - conçus comme «instruments de lutte contre la précarité [grâce à] une mutualisation des emplois au niveau des bassins d'emplois», des aides pour l'accès au logement et aux prêts bancaires pour les salariés titulaires d'un CDD, d'un temps partiel contraint et intérimaires, au moyen de fonds mutualisés. Le passage d'un temps partiel à un temps plein devrait également être facilité par des bourses d'emplois, instituées au niveau des branches professionnelles et/ou des bassins d'emplois.

Celles-ci devraient également se généraliser pour développer le reclassement externe en cas de licenciement économique: "Toutes les entreprises de la branche auraient l'obligation d'adresser leurs offres d'emploi à la bourse d'emploi mise en place. (...) La branche devrait négocier les modalités selon lesquelles s'opéreraient les mobilités interentreprises (maintien de rémunération, de l'ancienneté, aide au déménagement...) et les conditions de transférabilité des droits attachés au salarié (compte épargnetemps, formation...) d'une entreprise à l'autre et d'une branche à l'autre (fonds de péréquation)». La CFTC revendique par ailleurs un droit individuel au

(17) Audition de M. Jean-Claude Quentin, secrétaire confédéral chargé de l'emploi, de la formation professionnelle et de l'assurance chômage, M. Christophe Couillard et M. Youcef Tayeb, assistants confédéraux, CGT-FO, in CARLe, SeIllier, 2007, op. cit., Tome II, p. 131. 
reclassement pour tout salarié licencié pour raison économique, quelle que soit la taille de l'entreprise qui licencie. Elle propose un contrat entre le salarié et une structure chargée de gérer le dispositif, prévoyant notamment le maintien de la rémunération pendant douze mois. La rémunération et l'accompagnement individualisé dont bénéficierait le salarié seraient cofinancés par l'entreprise, le salarié, l'Assedic et l'AGS (Assurance garantie des salaires).

La CFTC insiste par ailleurs sur la nécessité de promouvoir le développement des régimes de prévoyance dans le cadre de la négociation d'entreprise ou de branche et propose d'étendre l'obligation annuelle de négocier sur le thème de la prévoyance maladie aux entreprises de moins de cinquante salariés (éventuellement dans le cadre de regroupement d'entreprises). En matière de droits à la retraite, le statut du travailleur propose d'une part la prise en compte de certaines périodes actuellement non validées (RMI, CES), sous condition d'engagement effectif dans un parcours d'insertion, et d'autre part l'ouverture de négociations de branches pour permettre au plus grand nombre de salariés à temps partiel de cotiser sur la base du salaire à temps plein pour améliorer leur retraite.

Enfin, le statut du travailleur prévoit des mesures visant à faciliter la conciliation entre vie personnelle, vie familiale et vie professionnelle. Le meilleur encadrement législatif du temps partiel, évoqué plus haut, en fait partie, mais aussi un meilleur encadrement des astreintes et des aménagements d'horaires.

\section{La CFE-CGC: accompagner les mobilités dans l'emploi, sécuriser les passages}

La CFE-CGC insiste sur l'importance de l'accompagnement des changements d'emploi dans l'entreprise, en privilégiant une logique préventive. Elle revendique ainsi un couplage entre la GPEC et le développement de compétences transférables par la formation continue, au profit de l'employabilité des personnes: dans la perspective de parcours professionnels continus, et indépendamment du lien privilégié avec une entreprise ou un emploi donné, «le concept de formation tout au long de la vie (...) mérite une actualisation de ses composantes que sont la transférabilité (18), l'obligation d'employabilité, la mutualisation, l'articulation entre les niveaux (national, régional...)» (LECANU,

(18) Concernant la notion de transférabilité, et la perspective en particulier d'un DIF transférable «dans la branche professionnelle et interbranches», la CFE-CGC indique: "conclure à la nécessité d'une gestion mutualisée, du type de celle en cours dans les Agefos, ne nous choquerait pas, à la condition d'une économie paritaire et suivie. En revanche, le problème du passage d'une branche à l'autre n'en serait pas résolu. L'existence du Fonds unitaire de péréquation constitue une piste», audition de M. Alain Lecanu, secrétaire national chargé du pôle emploi-formation, in CARLE, SeILlier, 2007, op. cit., Tome II, p. 215-217.
2006, p. 10). La CFE-CGC s'est ainsi prononcée pour une négociation nationale interprofessionnelle sur la GPEC, abordant également « la question territoriale, en particulier pour les TPE-PME(19)». L'objectif serait de rendre à la GPEC sa «finalité première: par une politique en amont, éviter les licenciements et non plus seulement accompagner les plans sociaux. C'est donc en veillant au maintien de l'employabilité et au développement des compétences des salariés que l'on sécurisera leur avenir professionnel et donc leur parcours» (ibid.). Tout en reconnaissant le caractère controversé de la notion d'employabilité, la CFE-CGC en fait «le chaînon manquant entre la flexibilité du travail et la sécurité de l'emploi, (...) un concept qui reste à traduire par des mesures concrètes » (CFE-CGC, 2003). La CFE-CGC considère par ailleurs qu'il convient aujourd'hui de travailler à la rénovation des concepts que sont: la relation de travail et les responsabilités des parties prenantes, le contrat de travail, l'organisation et les conditions de travail, la rémunération, le statut de l'encadrement (LeCANU, op. cit., p. 10).

S'agissant de la couverture par le régime d'assurance chômage, la CFE-CGC a proposé que l'ensemble des demandeurs d'emploi disposent d'un revenu de remplacement à hauteur quasiment de leur ancien salaire (ou d'un pourcentage du SMIC) pour une durée minimum de douze mois. Enfin, en terme de financement, la CGC a évoqué la création d'une taxe spécifique, afin que tous - y compris les fonctionnaires, professions libérales... - contribuent à «cet effort [de sécurisation des parcours professionnels] relevant de la responsabilité nationale et non plus uniquement interprofessionnelle $(20)$ ». Plus généralement, elle réaffirme aujourd'hui l'idée d'une "cotisation sociale sur la consommation» (LeCANu, op. cit., p. 10) pour renouveler les modalités de financement de la protection sociale.

Par ailleurs, dans le suivi et la sécurisation des parcours, la CFE-CGC est attentive aux nouvelles formes d'emploi qui se développent, notamment au portage salarial. Il constitue, pour la CGC, un montage juridique susceptible d'apporter un accompagnement, sur un temps limité, à un salarié engagé dans la création d'une activité indépendante. Les sociétés de portage, en tant qu'«intermédiaires de services $(21) \gg$ à des indépendants installés ou potentiels, peuvent participer à la réussite d'un projet de lancement d'une activité. Mais si le portage peut donc constituer un «sas de transition» (ibid.) entre

(19) «Déclaration du groupe de la CFE-CGC», in ConseIL ÉCONOMIQUE ET SOCIAL, 2007, op. cit., p. I-46.

(20) La newsletter de la CFE-CGC, mai 2005. http://www. cfecgc.org/TRANSFERT LIVE/Documents/AssChomageProp ositions052006.htm

(21) «Le portage salarial. Position de la CFE-CGC», décembre 2005. http://www.cfecgc.org/transfert/Documents \% 5CPortageSalarial122005.htm. 
une activité salariée et une activité d'indépendant, il ne peut, pour la CGC, devenir un mode durable et permanent de gestion de l'externalisation de salariés, en particulier des salariés âgés. Compte tenu des pratiques en vigueur, la CGC lui préfère aujourd'hui d'autres formes d'emploi - groupements d'employeurs ou entreprises de travail à temps partagé par exemple(22).

\section{L'UNSA: la progression professionnelle comme source de sécurisation}

L'intérêt communà l'ensemble du salariat est, pour l'UNSA, celui du développement de l'employabilité. En matière de reconversion et d'employabilité, l'UNSA insiste sur la responsabilité de l'entreprise. Elle revendique en particulier de créer un véritable droit à reconversion pour tous les salariés licenciés - et non plus pour les seuls salariés licenciés pour motif économique -, ainsi que l'instauration d' « une négociation dans l'entreprise autour de l'entretien professionnel, de la validation des acquis de l'expérience, de l'accès à la formation et de la reconnaissance des compétences » (UNSA, 2006, p. 15), en vue de préserver et d'accroître l'employabilité des salariés. Un certain nombre d'outils susceptibles de contribuer à la sécurisation des parcours existent d'ores et déjà dans 1'entreprise (entretien professionnel, GPEC(23), DIF), il conviendrait de les renforcer et d'en améliorer l'accès à tous les salariés pour construire des «parcours de qualification» (ibid., p. 16) dans l'entreprise. Si le salarié est de plus en plus acteur de sa qualification, l'employeur «a la responsabilité du développement de l'employabilité du salarié» (ibid.). L'UNSA précise par ailleurs qu'elle retient la définition de l'employabilité inscrite dans la recommandation 195 de l'OIT (24). Enfin, le contrat de travail, qui pour l'UNSA devrait être obligatoirement écrit, pourrait comporter, « outre la liste des stipulations juridiques ou conventionnelles, (...) un rappel des droits et obligations réciproques (25)».

(22) Créées par la loi du 2 août 2005 en faveur des PME.

(23) Concernant l'obligation de négocier sur la GPEC, l'UNSA revendique par ailleurs l'abaissement du seuil d'application à cinquante salariés et un soutien territorial pour les petites entreprises.

(24) «L'employabilité est encore un terme tabou dans le langage syndical, comme l'a été celui de compétence. Pour éviter tout faux débat de sémantique, l'UNSA propose de retenir la définition du Bureau international du travail inscrite dans la recommandation 195: "le terme employabilité se rapporte aux compétences et aux qualifications transférables qui renforcent la capacité d'un individu à tirer parti des possibilités d'éducation et de formation qui se présentent pour trouver un travail décent et le garder, progresser dans l'entreprise ou en changeant d'emploi, ainsi que s'adapter aux évolutions de la technologie et des conditions du marché du travail"» (UNSA, op. cit., p. 16). (25) UNSA, «Sécurisation des parcours professionnels. Extraits du texte voté», UNSA Magazine, $\mathrm{n}^{\circ}$ 90, septembreoctobre 2006, p. 11.
L'UNSA propose également la création d'un capital formation pour tous, droit différé à la formation attaché à chaque individu: un compte individuel de temps de formation (CITF) pourrait être créé, dont le capital de départ serait financé par les services publics de l'État ou des régions, que viendraient ensuite abonder les entreprises. En matière de formation professionnelle, l'UNSA plaide pour un droit universel à la formation transférable, au moyen de la création d'un «compte individuel de temps de formation, géré par un dispositif de mutualisation permettant la transférabilité du temps capitalisé en cas de mobilité externe à l'entreprise (26)». Enfin, l'UNSA plaide pour une réforme en profondeur du service public de l'emploi, qu'elle estime aujourd'hui «trop éclaté pour être vraiment efficace pour le demandeur d'emploi(27)». Réformer le SPE nécessite, selon l'UNSA, de créer un dispositif de guichet unique, seul susceptible d'offrir un réel accompagnement individualisé aux demandeurs d'emploi.

\section{Analyse sémantique}

Les positions syndicales, on le voit, semblent avoir eu tendance à se définir isolément, de façon autonome les unes par rapport aux autres, laissant à chacun le soin de s'orienter et de trouver les «clés » pour circuler entre des orientations et des présupposés pour partie distincts, mais qui en l'état restent encore difficiles à analyser. On peut cependant noter certaines constantes au plan sémantique: l'absence du terme «flexicurité» dans les corpus syndicaux au profit de celui de «sécurisation des parcours professionnels », la mobilisation par toutes les organisations de la notion de «transférabilité», l'usage le plus souvent prudent - mais différencié - du terme «employabilité». Revenons brièvement sur chacun de ces termes.

Les organisations syndicales françaises n'utilisent pas le terme de flexicurité, ou exceptionnellement, pour s'en démarquer. Le mot, à l'évidence, n'est pas neutre. Laurent Duclos et Jean-Yves Kerbourc'h notent, à cet égard, que «les réflexions sur la flexicurité (ont) trouvé leurs premiers terrains d'application dans les pays à tradition néocorporatiste »(Duclos, Kerbourc'H, 2006, op. cit., p. 11). Tros et WiLthagen (2004, p. 178) insistent sur cette corrélation entre politique de flexicurité et système de relations professionnelles. Tangian également, qui rappelle par ailleurs que la flexicurité, n'a pas pour but premier d'accroître la protection des travailleurs, mais vise l'atteinte d'un compromis entre employeurs et salariés, dans le cadre d'un

(26) «Déclaration du groupe de l'UNSA», in CONSEIL ÉCONOMIQUE ET SOCIAL, 2007, op. cit., p. I-66.

(27) «Les reconversions, éléments de sécurisation des parcours professionnels », UNSA Magazine, $\mathrm{n}^{\circ} 83$, janvier 2006, p. 18. 
trade-off entre flexibilité et sécurité (TANGian, 2005 , p. 10-11). La flexicurité viserait alors un type de compromis qui a pu être associé au «modèle» néocorporatiste. C'est sans doute l'une des raisons pour lesquelles le terme est, d'emblée, disqualifié en France. Les organisations syndicales françaises vont le remplacer par celui de sécurisation des parcours professionnels, en privilégiant l'idée de sécuriser les travailleurs précaires et ceux menacés dans leur emploi. La lutte contre toutes les formes de précarité de l'emploi - restructurations, éclatement des entreprises et des champs conventionnels, hétérogénéité et précarisation des statuts... - constitue en effet la visée commune, revendiquée par l'ensemble des organisations syndicales.

Plus controversé, entre les organisations syndicales, est le terme d' «employabilité». Il est, on l'a vu, explicitement mobilisé par deux organisations - la CFE-CGC et l'UNSA. Celles-ci prennent cependant le soin de signaler le caractère contesté de ce terme dans le monde syndical, preuve, s'il en était besoin, que l'enjeu sémantique est d'importance. On notera par ailleurs que le sens de ce terme reste relativement indéterminé du côté de la CFE-CGC. La majorité des organisations syndicales conserve donc un point de vue critique sur ce terme. Elles rejoignent en cela le point de vue également critique que Bernard Gazier développait en 1999, dans un rapport rendu à la Commission européenne(28). À ce jour, la plupart des organisations syndicales ont choisi de ne pas investir cette notion. Elles n'en développent pas de définition propre, considérant sans doute que le terme est trop manipulable par le patronat. Pour autant, le terme n'est pas absent des discussions avec le patronat. Il a notamment été utilisé lors des négociations de l'accord national interprofessionnel de 2003 sur la formation tout au long de la vie. Il resurgit d'ailleurs, à l'occasion, généralement avec des guillemets, lorsque l'une ou l'autre des organisations rappelle en particulier les objectifs de l'accord de 2003. Il n'en reste pas moins que les organisations syndicales ont un rapport difficile avec l'expression, compte tenu du déport implicite des responsabilités sur l'individu qu'elle suggère (29).

Enfin, si l'usage du terme «flexicurité» est pour partie «disqualifié», si le terme d' «employabilité» est prudemment tenu à distance, la «transférabilité»

(28) Pour une synthèse de ses conclusions sur la notion d'employabilité, voir GAZIER, 1999.

(29) La CFTC indique ainsi: «Nous percevons que (...) la réponse à la montée des incertitudes nouvelles ne peut pas se trouver uniquement dans des démarches individuelles, comme l'illustrent (par exemple) les interrogations sur (...) l'employabilité de chacun comme seule réponse au chômage» (CFTC, in CES, 2007, op. cit.). Signalons, pour la CFDT, la parution dans sa revue Cadres d'un article qui invite à substituer le terme de «recrutabilité» à la rhétorique de l'«employabilité», afin d'éviter de focaliser «sur un seul versant de la rencontre salariés-employeurs» (РосHIC, 2007). des droits est, quant à elle, une notion largement mobilisée dans les corpus syndicaux, en lien avec la notion de «mutualisation», partagée et revendiquée, elle aussi, par l'ensemble des organisations syndicales. Le glissement opéré, dans les corpus syndicaux, de l'idée de «droits attachés à la personne», à la notion de transférabilité des droits, au moyen de la technique de la mutualisation, suggère cependant une opposition entre la mutualisation - qui permet de garantir les droits au plan collectif - et l'individualisation des droits. L'enjeu de la gestion collective, dont on connaît l'importance, laisse en effet dans l'ombre la question des modes de gestion individualisés de ces droits, qui n'est pas directement abordée dans les écrits syndicaux sur la sécurisation des parcours professionnels, quoiqu'une référence y apparaisse à travers l'idée de droits attachés à la personne. Notons enfin que la question de la transférabilité des droits est également abordée comme un outil de lutte contre la précarité de l'emploi, permettant notamment de faire face aux situations de restructurations. L'enjeu alors, est bien de mutualiser également les obligations, en particulier en matière de reclassement (Duclos, KerbourC'H, 2006, op. cit., p. 76 et s; Chassard, Kerbourc'H, 2007, p. 1100).

Si les organisations d'employeurs ont formalisé plus tardivement leurs positions sur le thème de la flexicurité, notons ici qu'elles se sont positionnées sur la question de la transférabilité. Cette question était déjà présente lors des négociations, de 2001 à 2003, sur la formation professionnelle. La transférabilité du DIF, revendiquée par les organisations syndicales, avait alors été rejetée par la partie patronale. Medef et CGPME restent hostiles aujourd'hui à une «transférabilité généralisée» du DIF, considérant que celle-ci nécessiterait un provisionnement du droit qui viendrait grever les comptes des entreprises (30). Mais le Medef rappelle également que l'ANI de 2003 envisageait, dans certains cas, la possibilité d'un «abondement financier» (ibid.), à propos duquel les signataires de l'accord avaient sollicité «l'ouverture d'une concertation avec les pouvoirs publics». Nous n'entrerons pas, ici, dans le détail des propositions des organisations patronales. Notons simplement que, de façon générale, le Medef a principalement décliné l'idée de flexicurité autour de l'enjeu d'une «modernisation du droit des contrats de travail(31)»-introduction de nouveaux types de contrats «à rupture précausée» (ibid.), de nouveaux modes de rupture du contrat de travail.

(30) Audition de MM. Francis Da Costa, président de la commission formation, Bernard Falck, directeur de l'éducation et de la formation, Alain Druelles, chef du service formation professionnelle du Medef, in Carle, SeIllier, 2007, op. cit., p. 350-351.

(31) «Position du Medef», colloque «La sécurisation des parcours professionnels», CAS/COE, février 2007, p. 19-20. Texte extrait de Parisot, 2007, p. 19-20. 
La CGPME considère que le concept de sécurisation des parcours professionnels recouvre la nécessité de "procédures de licenciement suffisamment souples pour contribuer à la fluidité du marché du travail, mais aussi des mesures destinées à faciliter le reclassement des salariés licenciés de la façon la meilleure et la plus rapide (32)». Elle insiste sur le fait qu'il importe d'abord de «faire vivre pleinement les dispositifs existants » (ibid.) - contrat de transition professionnel et convention de reclassement personnalisé.

\section{Propositions syndicales et positions des organisations dans le champ des relations professionnelles}

Pour tenter de clarifier davantage les termes du débat, nous proposons de projeter sur une «carte» notre interprétation des différentes propositions syndicales, pour les mettre à distance, en comprendre mieux les ressorts et, dans l'espace ainsi formé, donner à penser. La mise en évidence des champs sémantiques privilégiés par chaque organisation suggère d'abord l'existence d'un lien fort entre ses propositions en matière de sécurisation des parcours professionnels, et sa position dans le système de relations professionnelles. En effet, les organisations syndicales ne se positionnent que dans le cadre institutionnel du travail salarié. Mais elles ont des préoccupations sur ce qui, le long de ce cadre, se craquelle, à proximité des frontières. La représentation graphique qui suit ( $c f$. figure 1) permet de mieux visualiser et analyser ces différentes dimensions des positions syndicales.

\section{La construction d'une "carte des positions syndicales"}

La «carte» formalisée ci-dessous repose sur le présupposé sociologique d'une «homologie structurale» (BouRdieU, 1987) entre les prises de position syndicales sur la sécurisation des parcours professionnels, et la position de chacune des organisations dans le champ des relations professionnelles: on peut en effet analyser les prises de position syndicales comme la rencontre entre, d'une part, un ensemble de solutions disponibles, de pratiques et de modèles possibles, produits par l'histoire antérieure de nos règles et de nos institutions et, d'autre part, les dispositions particulières des différentes organisations syndicales, ce à quoi elles sont prêtes. La façon dont les prises de position des organisations syndicales se distribuent, résulte alors de la mise en relation entre des prises de position possi-

(32) «Position de la CGPME», colloque «La sécurisation des parcours professionnels», CAS/COE, février 2007, p. 21. bles (propositions, pistes de solutions en matière de sécurisation) et des dispositions à emprunter ces pistes, à prendre ces positions. Ces dispositions sont étroitement liées aux positions qu'occupent aujourd'hui les organisations dans le champ des relations professionnelles. D'où le rapport d'homologie entre propositions syndicales sur la sécurisation des parcours et positions des organisations dans le système de relations professionnelles. Les «frontières » de la carte à dessiner sont celles du système de relations professionnelles. Elles sont aussi celles du droit du travail.

Nous empruntons donc à Alain Supiot le cadre des «frontières du travail salarié» (SUPIOT, 1995), au sein desquelles se déploie le système de relations professionnelles. Celles-ci sont délimitées par quatre oppositions, indique Supiot, qui permettent de situer le travail salarié dans un carré dont les côtés sont: l'opposition entre travail à titre onéreux et travail à titre gratuit, l'opposition entre travail salarié et fonction publique, l'opposition entre travail et formation professionnelle, l'opposition entre travail dépendant et travail indépendant. Cet espace, aux frontières de plus en plus poreuses, disait Supiot, est celui dans lequel s'organise le jeu du partenariat social institutionnalisé. C'est d'abord dans ce cadre structurel que peuvent s'analyser les propositions syndicales sur la sécurisation des parcours professionnels.

Puis, nous verrons que l'on peut également tenter de situer sur le schéma, dans un second temps, les deux «principes de recomposition du droit social» qu'évoque Supiot (1995, op. cit., p. 830), et dont il voit les prémisses dans les évolutions actuelles du droit positif: «pas de contrat sans statut», «pas de statut sans issue». Cela nous permettra d'interroger, au vu de la carte et des interprétations qu'elle suggère, la façon dont les organisations syndicales travaillent ces lignes de recomposition du droit social et les questions dont elles sont porteuses: comment donner un statut aux personnes dans l'exercice de leur profession et lequel («pas de contrat sans statut»)? Comment envisager les transitions et assurer la mobilité statutaire ( pas de statut sans issue »)?

On peut ainsi situer, dans l'espace formé par cette « définition institutionnelle du travail salarié» (SUPIOT, 1995, op. cit.,p. 829), chacune des organisations syndicales selon ce qui fait le cœur de ses propositions en matière de sécurisation des parcours professionnels, selon ce que sont ses principales préoccupations, ses «dispositions» propres. Les dispositions propres de la CGT peuvent être définies par deux choses: d'une part, l'état d'avancement, à ce jour, d'une solution ou d'un modèle possible - «l'horizon d'un nouveau statut pour le travail salarié», «l'élaboration possible d'une sécurité sociale professionnelle»-, solution et modèle qui, en présentant la possibilité effective de leur réalisation contribuent d'ailleurs à en susciter 
Figure 1

Propositions syndicales et positions des organisations dans le champ des relations professionnelles

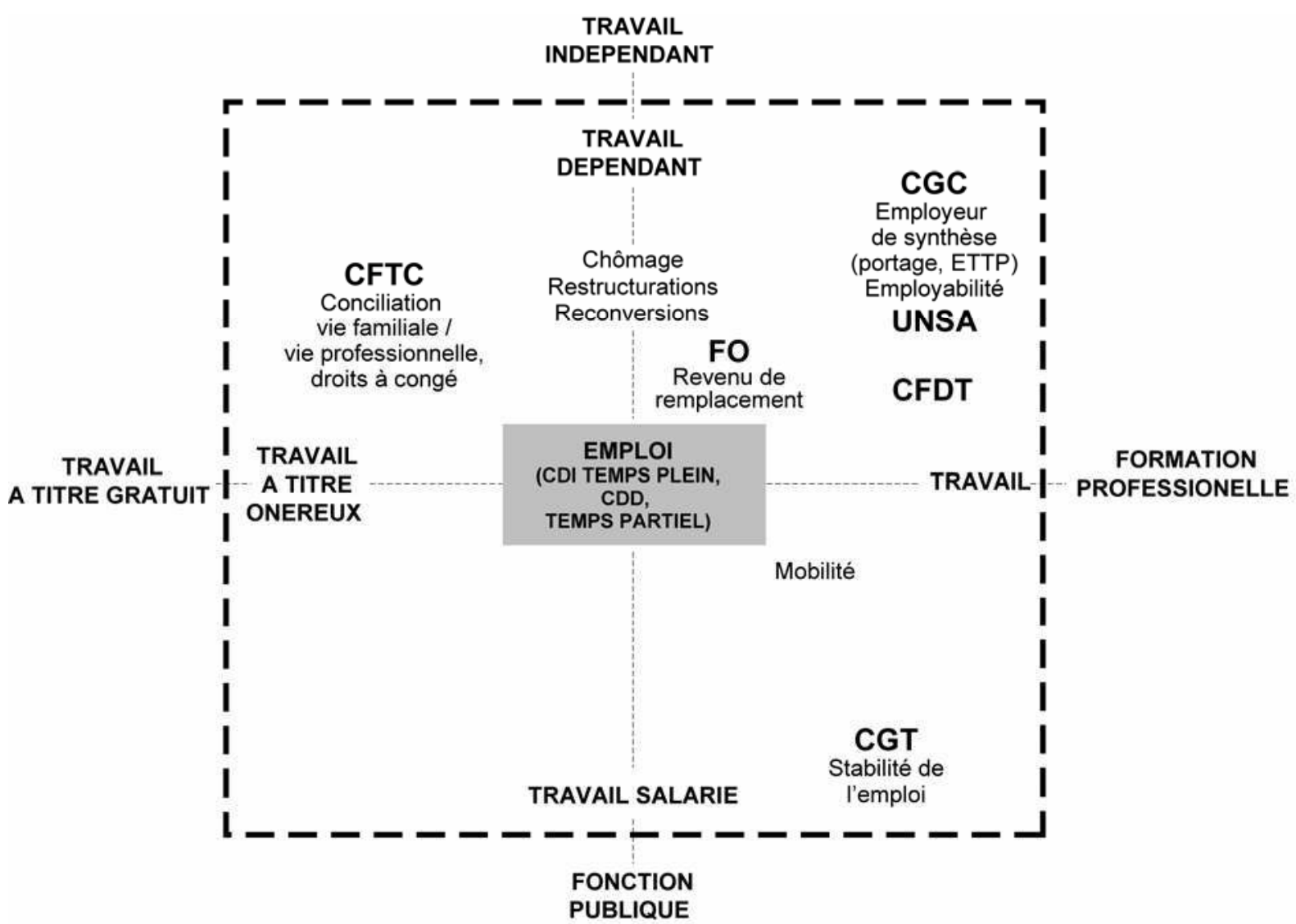

Les propositions des organisations syndicales s'inscrivent toutes dans le cadre institutionnel du travail salarié (le carré du schéma). Les deux lignes tracées en pointillé (au cœur du schéma) suggèrent, dans un second temps, comment les différentes organisations travaillent aujourd'hui, les questions que soulèvent les évolutions du droit positif : quelles sont les modalités possibles d'acquisition d'un statut dans le cadre de l'activité professionnelle (ligne verticale, «pas de contrat sans statut»); quelles sont les modalités de prise en charge des transitions et des passages entre différents statuts (ligne horizontale, «pas de statut sans issue»). Dans le premier cas, les notions et références mobilisées vont du statut de la fonction publique à celui d'indépendant, en passant par des dispositifs visant à organiser la continuité des droits sociaux (employeur de synthèse). Dans le second cas, les outils mobilisés vont des divers droits à congé aux dispositifs de formation professionnelle, en passant par le droit à la reconversion. Notons par ailleurs que l'absence de l'intérim dans la case centrale du schéma vient de ce que les modalités d'octroi d'un statut protecteur diffèrent dans ce cas, où pourvoyeur de travail (entreprise cliente) et pourvoyeur d'un statut d'emploi (entreprise de travail temporaire) sont disjoints.

le besoin; d'autre part, la mise en œuvre antérieurement à ces réflexions d'un certain «modèle social», dans lequel la CGT a pris sa part, dont la version la plus aboutie s'est sans doute incarnée, du point de vue de la CGT, dans le modèle de l'emploi public, et qui revêt pour cette confédération un sens particulier, associé de longue date à une certaine idée du progrès social que la CGT souhaite promouvoir (absence de rupture de la relation d'emploi, droit à la carrière notamment). On voit alors comment les «dispositions » dont fait preuve la CGT sur le thème de la sécurisation des trajectoires correspondent, ou font écho, à la position que cette organisation occupe plus généralement, et de longue date, dans le champ des relations professionnelles. La CGT-FO, quant à elle, fidèle à sa pratique contractuelle dans le champ des relations professionnelles, souhaitant dans cette perspective privilégier l'obtention d'avancées précises pour les salariés, au plan juridique, a d'abord montré une certaine réticence à propos de concepts qu'elle jugeait «trop flous » et «peu concrets »- sécurisation des parcours professionnels, formation tout au long de la vie. Par ailleurs l'intérêt historique que la CGTFO porte à l'Unedic - elle est directement à l'origine de la convention fondatrice de 1958(33) - la prédispose notamment à faire de l'assurance chômage et du revenu de remplacement l'un de ses principaux points d'entrée. Les organisations syndicales elles-mêmes, d'ailleurs, témoignent parfois de cette correspondance entre leurs propositions et la position qu'elles occupent dans l'espace des relations professionnelles: elles peuvent en effet donner à voir, dans la façon dont elles explicitent et justifient leurs choix, le système dans lequel elles s'inscrivent. Il arrive ainsi que les organisations s'adressent implicitement - ou

(33) Rappelons en effet que c'est la CGT-FO qui, en août 1957, prend l'initiative d'engager des discussions informelles avec le $\mathrm{CNPF}$ en vue d'aboutir à l'adoption d'un régime paritaire d'assurance chômage (DANIEL, TUCHSZIRER, 1999). 
explicitement - les unes aux autres. Lorsqu'elles le font, elles manifestent explicitement la «logique structurale» à l'intérieur de laquelle sont définies leurs propres pratiques, à travers laquelle s'effectuent leurs prises de position. C'est par exemple le cas lorsque la CFDT, se dissociant ainsi de la proposition de la CGT, indique que l'instauration de nouvelles garanties «ne relève pas de l'octroi unilatéral d'un statut, mais d'une construction progressive par la négociation collective et le compromis») (KARVAR, 2005, op. cit., p. 53).

Enfin, plus classiquement, le champ des relations professionnelles a également ses règles propres, et les organisations syndicales ont des intérêts qui sont également définis par la logique du jeu. Il existe en particulier des enjeux symboliques forts - conserver la présidence de l'Unedic, par exemple-, qui ne sont pas sans effet sur les positions adoptées - la CFDT est aujourd'hui naturellement portée à "s'inscrire [comme elle l'indique] dans les orientations de l'Unedic $»$. Il en découle que les prises de position des organisations syndicales sur le thème de la flexicurité sont les unes aux autres, ce que sont les unes aux autres les positions de ces mêmes organisations dans le champ des relations professionnelles. Les mêmes écarts de position, en particulier, subsistent. C'est en cela que l'on peut parler d'une forme d'homologie: les propositions de la CFDT en matière de "sécurisation des parcours professionnels», ou celles de la CFTC en matière de «sécurisation des parcours de vie», etc., sont à la «sécurité sociale professionnelle» de la CGT, ce que la CFDT ou la CFTC, etc., sont à la CGT dans l'espace des relations professionnelles.

Par ailleurs, on l'a dit, le schéma permet également d'interroger la façon dont chaque organisation syndicale travaille aujourd'hui sur les deux «principes de recomposition du droit social» qu'évoque Supiot - «pas de contrat sans statut», «pas de statut sans issue». Les organisations syndicales travaillent parfois de façon privilégiée l'un de ces deux principes - sans que cela soit pour autant à l'exclusion de l'autre. Le positionnement, sur la carte, des organisations selon la première dimension, celle des modalités d'octroi d'un statut, suggère de réelles différences : la CFTC inclut, par exemple, la situation des travailleurs indépendants pour concevoir son «Statut du travailleur», ce qui n'est pas le cas, comme son nom l'indique, du «Nouveau statut du travail salarié» de la CGT. Ce dernier, on l'a souligné, prolonge plutôt le modèle statutaire de la fonction publique. La CFE-CGC, sensible aux passages entre travail indépendant et travail salarié, se situe quant à elle, logiquement, plutôt vers le haut du schéma. Vraisemblablement, le positionnement des organisations selon ce premier «principe de recomposition" traduit également leur propension, ou non, à travailler le thème d'un possible «employeur de synthèse», et à s'intéresser aux dispositifs «capables d'assurer une partie des fonctions sociales de l'employeur, c'est-à-dire d'insti- tuer la continuité du statut face à la discontinuité des prestations de travail, (...) d'assurer la gestion du temps long de l'acquisition des droits sociaux face au temps court du contrat» (SUPIOT, 1995, op. cit., p. 830) (groupements d'employeurs par exemple). Dans cette perspective, la distribution des organisations semble ici témoigner de l'intérêt, diversement partagé, que celles-ci peuvent porter à la question de l'agencement de la «fonction employeur».

L'autre principe de recomposition - «pas de statut sans issue»-départage les organisations selon l'importance qu'elles donnent à la question de la mobilité statutaire et aux transitions, et selon les modalités qu'elles privilégient pour les prendre en charge. On retrouve ici les différents types de sécurité privilégiés par les organisations: conciliation entre vie familiale et vie professionnelle et exercice des droits à congés pour la CFTC; revenu de remplacement et droit à la reconversion pour FO, employabilité et mobilité statutaire pour la CGC; employabilité et formation pour l'UNSA; sécurité de l'emploi, assurance chômage, mobilité et formation pour la CFDT; stabilité de l'emploi et maintien du contrat de travail enfin pour la CGT. C'est pour partie le long de cette «ligne» que les organisations sont amenées à travailler la question de nouveaux droits (congés, reconversion, formation initiale et continue, qualification...) et de leurs modalités d'exercice. Cela les conduit également, dans certains cas, à évoquer la question du territoire, bien que celle-ci soit encore souvent traitée de façon relativement floue ou «connexe», par des organisations syndicales qui restent, en pratique, encore très centrées sur la branche et l'interprofessionnel. Il y a certainement là un enjeu essentiel en matière de sécurisation des parcours professionnels. Le récent avis du CES insiste également sur ce point. Si le territoire, en effet, est le plus souvent l'échelle des souhaits et des possibilités de mobilité des salariés, il est aussi, considère Bernard Gazier, le lieu possible de négociations, susceptibles de déboucher sur des formes de «sécurisation active et de long terme» (GAZIER, 2000, p. 577-578).

\section{* *}

$*$

Le thème de la flexicurité ne semble donc toujours pas, à ce jour, collectivement problématisé(34): le cheminement nécessaire à la réflexion collective pour le traiter ne semble pas, en effet, claire-

(34) En dépit de l'avancée des travaux du groupe «Sécurisation des parcours professionnels» du Conseil d'orientation pour l'emploi (mai 2007), et de l'avis du CES sur la «Sécurisation des parcours professionnels» (2007). Ce dernier a notamment été l'occasion, pour les organisations syndicales, de retravailler leurs propres positions, de les préciser tout en maintenant leurs spécificités, et de formuler dans bien des cas des réserves sur le texte de l'avis, sur des points qui ne sont pas toujours mineurs. 
ment balisé. Cette difficulté à problématiser découle notamment d'un déficit initial de concertation, qui n'a pas été véritablement démenti ensuite. La difficulté à problématiser sur le plan collectif n'est sans doute pas pour rien dans le fait que les débats plus récents se sont pour partie focalisés sur le contrat de travail: en l'absence de ce travail de problématisation, et d'un véritable processus de concertation, les rapports publics tendent, aujourd'hui encore, à surdéterminer les termes du débat. Le rapport CAHUCKRAMARZ (2004) aura ainsi fait du contrat unique un point de passage obligé. Les travaux du Conseil d'orientation pour l'emploi sur la sécurisation des parcours professionnels se sont eux aussi concentrés, à leurs débuts du moins(35), sur la question du contrat de travail. Le cycle de «délibérations sociales» convoqué ensuite par le Medef témoigne, notamment, de ce que le partenariat social, depuis le projet de «refondation sociale» du Medef en 1999, est en quête de nouvelles dénominations susceptibles de qualifier les pratiques de dialogue social (DucLos, Mériaux, 2001, p. 19-34). L'intitulé semble être aujourd'hui le symptôme d'une difficulté à articuler la négociation en tant que décision, et la négociation en tant que délibération, faisant surgir son objet. Le terme de "délibération», en effet, renvoie a priori à autre chose qu'à une "stricte négociation", en tant que cette dernière s'inscrit avant tout dans une trame exécutive. La délibération, à l'instar de la concertation, devrait notamment permettre l'émergence de nouveaux problèmes (Duclos, MiRANDON, op. cit., 2003), constituer un espace sur fond duquel pourrait s'établir, ensuite, une négociation(36). Autrement dit, la délibération a vocation à produire des objets pour la négociation, qui, elle, produit des décisions à effet de droit. Cette distinction peut passer inaperçue tant que l'on s'en tient aux champs habituels de la négociation: autant, sur la question des salaires, il n'y a pas de problème de détermination de l'objet de la négociation, autant on est, sur la sécurisation des parcours professionnels, dans une thématique plus floue, qui ne constitue pas en tant que telle un objet de négociation. Mais le thème, tel qu'il est décliné à ce jour, est-il à la mesure de l'enjeu de la sécurisation des parcours professionnels? N'y a-t-il pas, notamment, le risque d'une forme d'enfermement des discussions autour du contrat de travail? Laurent Duclos et Jean-Yves Kerbourc'h considèrent ainsi que l'un des enjeux en matière d'organisation du marché du travail et de flexicurité est précisément de sortir d'un enfermement dans le pôle du contrat de travail, et de "faire de la "trajectoire" l'instance commandant la combinaison des protections plutôt

(35) Le rapport d'étape provisoire (mai 2007) du groupe «Sécurisation des parcours professionnels» du COE insiste surtout sur les limites du contrat unique.

(36) Notons sur ce point que l'avis du CES souligne la nécessité de laisser le temps utile à la concertation et la négociation sur ce sujet. que d'attacher chacune de ces protections à un état contingent. Il faudrait donc pouvoir déconnecter les engagements et les contrats de la question des droits sociaux, majoritairement acquis aujourd'hui au titre d'un engagement ou d'un contrat défini, établis qui plus est dans un champ conventionnel déterminé» (Duclos, Kerbourc'H, 2006, op. cit., p. 67). Il s'agirait, autrement dit, de « concevoir de nouvelles formes d'universalité des droits, c'est-à-dire des droits qui peuvent s'acquérir et être consommés en dehors du cadre de référence de l'entreprise» (ibid., p. 69). Par ailleurs, le risque d'une forme d'enfermement dans le thème du contrat de travail ne vient-il pas, pour une part, d'une tendance - renouvelée? - au paternalisme qui, comme le rappelle un rapport récent de l'OIT, devrait pourtant être considéré "comme une forme d'insécurité et comme un mécanisme pour susciter un sentiment d'insécurité(37)».

Enfin, la «carte» que nous avons proposée suggère une difficulté: si les positions des uns et des autres se présentent comme des sortes de «blocs de positions », il devient plus difficile de considérer ce qui surgit entre les positions établies, qui est peu abordé, voire pas toujours nommé. Certains auteurs avancent, de ce point de vue, d'autres questionnements ou pistes de solution. Marie-Laure Morin interroge par exemple le rôle du «réseau d'entreprise ou [du] tiers employeur: sont-ils des employeurs (ou plutôt des co-employeurs) au sens juridique du terme, ou ne sont-ils que des intermédiaires qui organisent la circulation entre différents employeurs? Quelle est alors leur responsabilité propre?» (MoRIN, 2003, p. 109). D'autres notions existent, telles que par exemple l'employeur de transition(38), qui remettent au centre des débats la question de l'articulation entre marché du travail et protection sociale au sens large, question qui était d'ailleurs au cœur des premières réflexions syndicales sur la sécurisation des trajectoires professionnelles (Le Duigou, 2005; Karvar, 2005, op. cit.). C'est bien l'un des enjeux d'une problématisation collective du thème de la flexicurité.

(37) BIT, 2005, p. 8: «Certains observateurs évoquent l'existence de modèles de sécurité dépendante, laquelle est proche du paternalisme. Ce n'est pas la sécurité réelle (...). Le paternalisme a souvent de bonnes intentions. Par exemple, on parle souvent de "droits assortis de responsabilités" pour justifier l'imposition de conditions aux plus démunis pour qu'ils bénéficient du soutien de l'État. Le problème, c'est que la nature directive de la "responsabilité" imposée soustrait effectivement le bénéficiaire du domaine de la responsabilité ou de la liberté pour le placer dans le domaine de l'obligation. Le paternalisme bien intentionné se confond aisément avec la coercition discrétionnaire et arbitraire. Lorsque les dirigeants et les élites de la nation essaient d'imposer leur vision de la "vertu", ceux qui voient le monde différemment et veulent vivre selon leur propre code de vertu se retrouvent dans une situation de plus grande insécurité, voire marginalisés ou isolés. L'imposition d'une norme sociétale - aussi bonne et noble soit-elle - est un moyen de faire vivre les hommes dans la peur. Le paternalisme n'est pas une solution à l'insécurité».

(38) $C f$. les propositions du rapport Belorgey du Commissariat GÉNÉRAL DU PLAN (2000). 


\section{Bibliographie}

BIT (2005), La sécurité socio-économique pour un monde meilleur, Genève.

Boccara P. (1995), «Sur de nouveaux principes et institutions mixtes pour la création d'emplois et l'insertion dans un autre plein emploi et une pleine activité», in "Pour une sécurité d'emploi ou de formation », ISSUES, $\mathrm{n}^{\circ} 47$ $48,4^{\mathrm{e}}$ trimestre $1995-1^{\mathrm{er}}$ trimestre 1996 , p. 9-105.

Bourdieu P. (1987), Choses dites, Paris, Les Éditions de Minuit.

Cahuc P., Kramarz F. (2004), De la précarité à la mobilité: vers une Sécurité sociale professionnelle, Paris, La Documentation française.

CARle J.-P. (président), SeIllier B. (rapporteur) (2007), Rapport de la mission commune d'information sur le fonctionnement des dispositifs de formation professionnelle, Sénat, Rapport d'information no 365 , juillet.

Cerc (2005), La sécurité de l'emploi. Face aux défis des transformations économiques, Paris, La Documentation française.

CFE-CGC (2003), «Employabilité, le chaînon manquant», Encadrement Magazine, n 107, septembre.

CGT (2003), «Nouveau statut du travail salarié», Document de travail, $\mathrm{n}^{\circ} 3$, février.

CGT (2007), «Négociation sur le marché du travail. Au pas de charge », La Nouvelle Vie Ouvrière, 26 octobre.

Chassard Y., Kerbourc’h J.-Y. (2007), « Négociation sur la modernisation du marché du travail: ne pas se tromper d'époque», Droit Social, no 11, novembre, p. 10951104.

Commissariat général du Plan (1995), Le travail dans vingt ans, Rapport de la commission Boissonnat, Paris, Odile Jacob.

COMmissariat GÉNÉral du Plan (2000), Minima sociaux, revenus d'activité, précarité, rapport du groupe présidé par J.-M. Belorgey, Paris, La Documentation française, mai.

Commissariat général du Plan (2000), Rapport sur les perspectives de la France, Paris, La Documentation française.

Conseil ÉCONOMIQUe ET SOCIAL (2007), «La sécurisation des parcours professionnels », Avis sur le rapport présenté par $\mathrm{M}^{\mathrm{me}}$ E. Arnoult-Brill au nom de la section du travail, Les Éditions des Journaux Officiels, juin.

CONSEIL D'ORIENTATION POUR L'EMPLOI (2007), Rapport d'étape sur la sécurisation et la dynamisation des parcours professionnels, mai.

Daniel C., Tuchszirer C. (1999), L'État face aux chômeurs. L'histoire de l'indemnisation du chômage de 1884 à nos jours, Paris, Flammarion.

Donneddu J. (2006), «Assurance chômage: quelles garanties collectives et protection sociale des salariés?», Analyses \& Documents économiques, $\mathrm{n}^{\circ}$ 101-102, avril.

Duclos L., Mériaux O. (2001), «Autonomie contractuelle et démocratie sociale: les implicites de la "refondation" ", Regards sur l'actualité, janvier, p. 19-34.

Duclos L., Mirandon M. (2003), «Le mandant du Plan, mutatis mutandis», Document d'étude, Commissariat général du Plan, service des affaires sociales, février.

Duclos L., Kerbourc'H J.-Y. (2006), Organisation du marché du travail et flexicurité à la française, Rapport pour le Conseil d'orientation pour l'emploi, novembre.

Dumas M. (2002), «Formation et nouveau statut du travail salarié: un enjeu décisif», Analyses \& Documents économiques, $\mathrm{n}^{\mathrm{0}}$ 91, octobre, p. 38-40.

ForCE OUVRIÈRE (2005), «Cohésion sociale \& accompagnement des mutations économiques», Bulletin Emploi, $\mathrm{n}^{\mathrm{o}} 4$, janvier.

Friot B. (1998), Puissances du salariat. Emploi et protection sociale à la française, Paris, La Dispute.

GABRIEl J.-P. (2006), «Nouveau statut du travail salarié et Sécurité sociale professionnelle: les propositions de la CGT», Communication au séminaire MESPI, Paris, 30 mai.

GAzIER B. (1999), «Assurance chômage, employabilité et marchés transitionnels du travail», Cahiers de la MSE, $\mathrm{n}^{\circ} 9903$.

GAZIER B. (2000), «L'articulation justice locale/justice globale. Le cas des "marchés transitionnels du travail"», Revue économique, vol. 51, $\mathrm{n}^{\mathrm{O}} 3$, mai.

Karvar A. (2005), «Face à l'insécurité sociale. Imaginer de nouvelles garanties », Cadres-CFDT, n ${ }^{\circ} 414$, avril.

Le Duigou J.-C. (2002), «Approche économique d'une sécurité sociale professionnelle. Contexte et contenu», Analyses et Documents économiques, $\mathrm{n}^{\circ} 89$, janvier.

Le Duigou J.-C. (2005), «La sécurité sociale professionnelle. Une utopie réaliste», Analyses \& Documents économiques, $\mathrm{n}^{\circ} 98$, février, p. 44-49.

Le Duigou J.-C. (2007), «D'un nouveau plein-emploi à la démocratie sociale», L'Économie politique, $\mathrm{n}^{\mathrm{0}} 34$, avril.

Lecanu A. (2006), «Comment sécuriser les parcours professionnels: l'assurance emploi», Encadrement Magazine, $\mathrm{n}^{\circ} 136$, juillet-août.

Morin M.-L. (2003), «Le droit du travail à l'épreuve de la "flexicurité", questions et perspectives d'analyse», in "Souplesse et sécurité de l'emploi: orientations d'études et recherches à moyen terme», Document d'études, Dares, Carole Yerochewski (coord.), no 71, juin.

PArisot L. (2007), Besoin d'air, Paris, Éditions du Seuil, février. 
Pochic S. (2007), «Employabilité ou recrutabilité?», Cadres-CFDT, no 524-426, août, p. 27-34.

Quentin J.-C. (2006), «DIF : ne vous laissez pas impressionner!», FORMPRO, CGT-FO, n 38 , septembre.

SimON G. (dir.) (2006), Le statut du travailleur. La sécurisation des parcours de vie, CFTC, avril.

Supiot A. (dir.) (1999), Au-delà de l'emploi. Transformations du travail et devenir du droit du travail en Europe, Rapport pour la Commission européenne, Paris, Flammarion.

Supiot A. (1995), «L'avenir d'un vieux couple: travail et Sécurité sociale», Droit Social, no 9-10, septembreoctobre, p. 823-831.
TANGIAN A.S. (2005), «Monitoring flexicurity policies in the EU with dedicated composite indicators", WSI diskussionspapier Nr. 137, June 2005, Düsseldorf, Hans Böckler Stiftung, p. 10-11. Invited paper presented at the DG Employment, Social Affaires and Equal Opportuinities, European Commission, Brussels, on March 18.

UNSA (2006), «Sécurisation des parcours professionnels », UNSA Magazine, $\mathrm{n}^{\circ} 83$, janvier.

Wilthagen T., Tros F. (2004), «The concept of 'flexicurity': a new approach to regulating employment and labour markets », Transfer, $\mathrm{n}^{\circ} 2$. 